\title{
SECURE VICKREY AUCTIONS WITHOUT A TRUSTED THIRD PARTY
}

\section{A Prototype}

\author{
Bart De Decker \\ K.U.Leuven, Dept. Computer Science, DistriNet, \\ Celestijnenlaan 200A, 3001 Heverlee, Belgium \\ Bart.DeDecker@cs.kuleuven.ac.be
}

\author{
Gregory Neven* \\ Gregory.Neven@cs.kuleuven.ac.be
}

\author{
Frank Piessens \\ Frank.Piessens@cs.kuleuven.ac.be
}

\begin{abstract}
Secure distributed computing addresses the problem of performing a computation with a number of mutually distrustful participants, in such a way that each of the participants has only limited access to the information needed for doing the computation. Over the past two decades, a number of solutions requiring no trusted third party have been developed using cryptographic techniques. The disadvantage of these cryptographic solutions is the excessive communication overhead they incur. In this paper, we examine a case study: a Vickrey auction without a trusted third party. The design is explained and the communication overhead studied. Several techniques to reduce or alleviate this overhead are discussed.
\end{abstract}

Keywords: Secure Vickrey Auction, Secure Distributed Computing, SDC, Agents, Multiparty computation

*Research Assistant of the Fund for Scientific Research - Flanders, Belgium (F.W.O.)

The original version of this chapter was revised: The copyright line was incorrect. This has been corrected. The Erratum to this chapter is available at DOI: 10.1007/978-0-387-35691-4_52 


\section{Introduction}

Most auctions are organized as follows: participants gather at a certain date and place, and make bids for a certain good. When the auction closes, the participant with the highest bid receives the good at the offered price.

Vickrey auctions differ in two essential points from traditional auctions:

- the participants deliver their bid in a sealed envelope to the bailiff; hence, the bid is not disclosed to the other participants. When the auction closes, the bailiff opens the envelopes and announces the winner.

- the winner (the participant with the highest bid) will have to pay the second highest bid.

The bailiff is the trusted third party (TTP). He should enjoy the confidence of every participant. The paper deals with a Vickrey auction ${ }^{1}$ without a TTP.

The structure of this paper is as follows. In the next section, we start with a brief introduction to the secure distributed computing problem (SDC). In section 2, we show how SDC can be used to organize a Vickrey auction without a trusted third party. We discuss the design of the boolean circuit that implements the auction. The experimental results are discussed in section 3. Especially the communication overhead is assessed and optimizations are proposed in section 4. Finally, in section 5, we summarize the major outcomes of this article.

\section{Secure Distributed Computing}

Secure distributed computing (SDC) addresses the problem of distributed computing where some of the algorithms and data that are used in the computation must remain private. Usually, the problem is stated as follows, emphasizing privacy of data. Let $f$ be a publicly known function taking $n$ inputs, and suppose there are $n$ parties (named $\left.P_{i}, i=1 \ldots n\right)$, each holding one private input $x_{i}$. The $n$ parties want to compute the value $f\left(x_{1}, \ldots, x_{n}\right)$ without leaking any information about their private inputs (except of course the information about $x_{i}$ that is implicitly present in the function result) to the other parties. An example

\footnotetext{
${ }^{1}$ Although Vickrey auctions may not seem very important, small variations on this scheme are used by many public institutions: e.g., a call for tenders, where the contract is given to the lowest offer (or the second lowest).
} 
is voting: the function $f$ is addition, and the private inputs represent yes $\left(x_{i}=1\right)$ or no $\left(x_{i}=0\right)$ votes. In case an algorithm has to be kept secret, instead of data, $f$ can be an interpreter for some (simple) programming language, and one of the $x_{i}$ can be the encoding of the algorithm.

In descriptions of solutions to the secure distributed computing problem, the function $f$ is often encoded as a boolean circuit, and therefore secure distributed computing is also referred to as secure circuit evaluation.

It is easy to see that an efficient solution to the secure distributed computing problem would be an enabling technology for a large number of interesting distributed applications across the Internet. Some example applications are: auctions ([15]), charging for the use of algorithms on the basis of a usage count ([16-17]), various kinds of weighted voting, protecting mobile code integrity and privacy $([17,12]), \ldots$

Secure distributed computing is trivial in the presence of a globally trusted third party(TTP): all participants send their data and code to the TTP (over a secure channel), the TTP performs the computation and broadcasts the results. The main drawback of this approach is the large amount of trust needed in the TTP.

However, solutions without a TTP are also possible. Over the past two decades, a fairly large variety of solutions to the problem has been proposed. An overview is given by Franklin [8] and more recently by Cramer [5] and Neven [14]. These solutions differ from each other in the cryptographic primitives that are used, and in the class of computations that can be performed (some of the solutions only allow for specific kinds of functions to be computed). The main drawback of these solutions is the heavy communication overhead that they incur.

In [9], Franklin and Haber propose a protocol for secret circuit evaluation. The boolean circuit is evaluated on encrypted data with a homomorphic probabilistic encryption scheme.

- An encryption technique is probabilistic if the same cleartext can encrypt to many different ciphertexts. To work with encrypted bits, probabilistic encryption is essential, otherwise only two ciphertexts (the encryption of a zero and the encryption of a one) would be possible, and cryptanalysis would be fairly simple.

- An encryption technique is homomorphic if it satisfies equations of the form $E(x \mathbf{o p} y)=E(x) \mathbf{o p}^{\prime} E(y)$ for some operations op and $\mathbf{o p}^{\prime}$.

Recently, Cramer, Damgard and Nielsen have proposed in [6] a similar protocol that requires fewer encrypted bits per AND-gate. These optimizations are applied in the description of the Franklin-Haber protocol. 
The modified Franklin-Haber protocol. The protocol is based on a joint encryption scheme. All participants know the public key $K_{p u b}$ while each participant $P_{1}, \ldots, P_{n}$ has his own private key $K_{1}, \ldots, K_{n}$. Using the public key, anyone can create an encryption $E_{S}(m)$ of some message $m$, where $S \subseteq\left\{P_{1}, \ldots, P_{n}\right\}$, such that the private key of each participant in $S$ is needed to decrypt the message. More formally, if $D_{i}$ denotes the decryption with $P_{i}^{\prime}$ 's private key, the relation between encryption and decryption is given by: $D_{i}\left(E_{S}(m)\right)=E_{S \backslash\left\{P_{i}\right\}}(m)$. The plaintext $m$ should be easily recoverable from $E_{\emptyset}(m)$.

In the joint encryption scheme used by Franklin and Haber [9], a bit $b$ is encrypted as

$$
E_{S}(b)=\left[g^{r} \bmod N,(-1)^{b}\left(\prod_{j \in S} g^{K_{j}}\right)^{r} \bmod N\right]
$$

where $N=p q, p$ and $q$ are two primes such that $p \equiv q \bmod 4$, and $r \epsilon_{R}$ $Z_{N}$. The public key is given by $\left[N, g, g^{K_{1}} \bmod N, \ldots, g^{K_{n}} \bmod N\right]$. This scheme has some additional properties that are used in the protocol:

- XOR-Homomorphic. Anyone can compute a joint encryption of the XOR of two jointly encrypted bits. Indeed, if $E_{S}(b)=[\alpha, \beta]$ and $E_{S}\left(b^{\prime}\right)=\left[\alpha^{\prime}, \beta^{\prime}\right]$, then $E_{S}\left(b \oplus b^{\prime}\right)=\left[\alpha \alpha^{\prime} \bmod N, \beta \beta^{\prime} \bmod N\right]$.

- Blindable. Given an encrypted bit, anyone can create a random ciphertext that decrypts to the same bit. Indeed, if $E_{S}(b)=[\alpha, \beta]$ and $r \in_{R} Z_{N}$, then

$$
\left[\alpha g^{r} \bmod N, \beta\left(\prod_{j \in S} g^{K_{j}}\right)^{r} \bmod N\right]
$$

is a encryption of the same bit.

- Witnessable. Any participant can withdraw from a joint encryption by providing the other participants with a single value. Indeed, if $E_{S}(b)=[\alpha, \beta]$, it is easy to compute $D_{i}\left(E_{S}(b)\right)$ from

$$
W_{i}([\alpha, \beta])=\alpha^{-K_{i}} \bmod N
$$

First of all, the participants must agree on a value for $N$ and $g$, choose a secret key $K_{i}$ and broadcast $g^{K_{i}} \bmod N$ to form the public key. To start the actual protocol, each participant broadcasts a joint encryption of his input bits.

For an XOR-gate, every participant simply applies the XOR-homomorphism. The encrypted output of a NOT-gate can be found by applying the XOR-homomorphism with a default encryption of a one, e.g. 
$[1,-1]$. However, it is the AND-gate that causes some trouble. Suppose the encrypted input bits for the AND-gate are $\hat{u}=E(u)$ and $\hat{v}=E(v)$. To compute a joint encryption $\hat{w}=E(w)=E(u \wedge v)$, they need each others' help. The protocol proceeds as follows [6]:

1 Each $P_{i}$ chooses a random bit $b_{i}$ and broadcasts $\hat{b}_{i}=E\left(b_{i}\right)$.

2 Each participant repeatedly applies the XOR-homomorphism to calculate $\hat{u}^{\prime}=E\left(u^{\prime}\right)=E\left(u \oplus b_{1} \oplus \ldots \oplus b_{n}\right)$ from the received values of the first step. Each participant broadcasts a decryption witness for $\hat{u}^{\prime}$, i.e. $W_{i}\left(\hat{u}^{\prime}\right)$.

3 Everyone can now decrypt $\hat{u}^{\prime}$ into $u^{\prime}$. Next, every partipant can calculate $\hat{w}^{\prime}=E\left(u^{\prime} \wedge v\right)=E\left(\left(u \oplus b_{1} \oplus \cdots \oplus b_{n}\right) \wedge v\right)$.

If $u^{\prime}$ is 0 , then $u^{\prime} \wedge v=0$, and $E\left(u^{\prime} \wedge v\right)=E(0)$. However, if $u^{\prime}$ is 1 , then $u^{\prime} \wedge v=v$, and $E\left(u^{\prime} \wedge v\right)=E(v)$.

4 Every participant $P_{i}$ calculates and broadcasts $\hat{w}_{i}=E\left(b_{i} \wedge v\right)$. Since $P_{i}$ chose $b_{i}$, he knows the outcome of $b_{i} \wedge v$ (either 0 or $v$ ). Note that when $b_{i}$ is 1 , the participant should blind $E(v)$, thus ensuring that $b_{i}$ remains secret to the other participants.

5 Every participant repeatedly applies the XOR-homomorphism to calculate $\hat{w}=E(w)=E\left(w^{\prime} \oplus w_{1} \oplus \cdots \oplus w_{n}\right)$ from the values received in the previous step. Note that $\hat{w}$ is an encryption of wanted $u \wedge v$. Indeed:

$\begin{array}{rlclllll}w & = & u & & & & & \\ & = & \left(u \oplus b_{1} \oplus \cdots \oplus b_{n}\right. & \oplus & b_{1} & \oplus \cdots \oplus & \left.b_{n}\right) & \wedge v \\ & = & \left(u^{\prime}\right. & \oplus & b_{1} & \oplus \cdots \oplus & \left.b_{n}\right) & \wedge v \\ & = & \left(u^{\prime} \wedge v\right) & \oplus & \left(b_{1} \wedge v\right) & \oplus \cdots \oplus & \left(b_{n} \wedge v\right) & \\ & = & w^{\prime} & \oplus & w_{1} & \oplus \cdots \oplus & w_{n} & \end{array}$

When all the gates in the circuit have been evaluated, every participant has a joint encryption of the output bits. Finally, all participants broadcast decryption witnesses to reveal the output ${ }^{2}$.

In this protocol, the evaluation of an AND-gate requires that every participant broadcasts two encrypted bits $\left(E\left(b_{i}\right)\right.$ and $\left.E\left(b_{i} \wedge v\right)\right)$ and one decryption witness (which is half the size of an encrypted bit); hence, the total communication overhead amounts to 2.5 encryption bits per AND-gate (4 encryption bits in the original protocol [9]).

\footnotetext{
${ }^{2}$ Note that at this stage, some of the participants might try to cheat and broadcast wrong witnesses or broadcast nothing at all. However, this is noticeable to the honest participants.
} 


\section{Design of a Vickrey Auction without TTP}

We assume that there are $n$ bidders. The item will only be sold if the bid of one participant is strictly higher than the other bids. In all other cases there is no wimner. The clearing price is the second highest bid. The requirements for this type of auction are the following:

- if there is no wimner, nothing else (but the fact that there is no winner) is revealed;

- if there is a winner:

- reveal the identity of the highest bidder, but hide the highest bid;

- reveal the $2^{\text {nd }}$ highest bid, but hide the identity of the $2^{\text {nd }}$ highest bidder;

- do not reveal any other information.

In the modified Franklin and Haber protocol, the participants evaluate a boolean circuit on enciphered data; the data being the participant's bid. Hence, the first task is to design a boolean circuit that implements these requirements.

\subsection{Boolean Circuit for the Auction}

For three participants $x, y$ and $z$, the boolean circuit is shown in figure 1 . The inputs to the circuit are $b$-bit bids $(X, Y$ and $Z)$. The output is the identity of the winner, represented by the bits $R 1$ and $R 0$ $(R 1 R 0=00$ no winner, 01 winner is $x, 10$ winner is $y, 11$ winner is $z)$, and the clearing price. If there is no winner, the clearing price is set to zero. To determine the winner, the circuit uses three comparators and a number of AND and OR gates. To determine the clearing price, four multiplexers are used. Consider the situation where $X$ makes the highest bid. In this case $G 1 \wedge G 2=1, L 1 \wedge G 3=0, L 2 \wedge L 3=0$ and $R 1 R 0=10$, so the second input to the final multiplexer will be chosen. The input on this line is determined by the bids made by $y$ and $z$. If $Y>Z$ then $G 3=1$ and $Y$ will be selected as the clearing price. In the other cases $(Y<Z$ or $Y=Z) Z$ will be the clearing price.

It is rather straightforward to extend the circuit for more than three participants.

The circuits for comparators and multiplexers are designed in such a way that the number of AND-gates are minimized. OR-gates can be transformed into AND-gates using the "De Morgan" laws. Figure 2 illustrates the circuit for a comparator that compares two 2-bit numbers. 


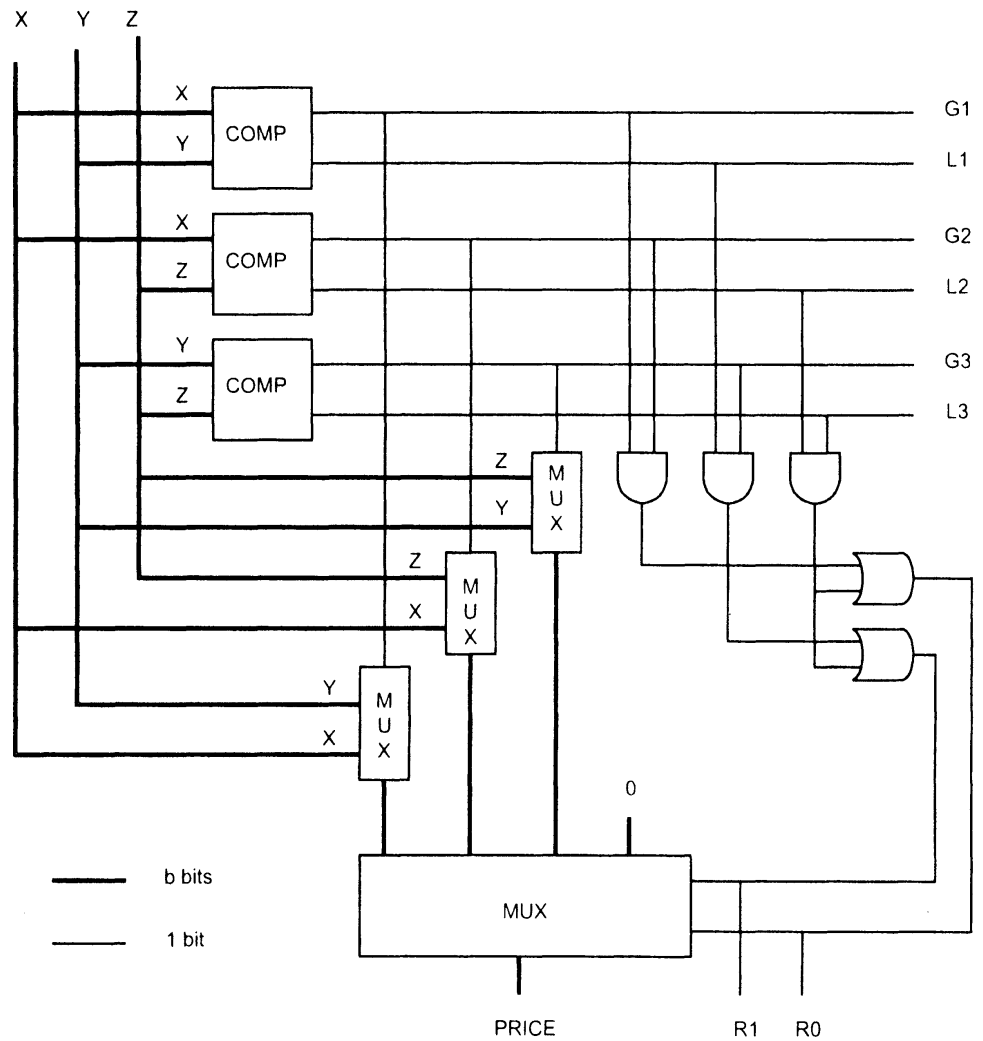

Figure 1. Boolean circuit of the Vickrey Auction for 3 bidders.

It is not difficult to extend the circuit for longer numbers. However, there is no general design strategy for minimizing the number of AND-gates.

\section{$2.2 \quad$ The prototype}

The prototype has been written in Java. All bidders in the auction run as a separate thread within one Java application. Broadcast communication was simulated through the use of shared buffers.

In all experiments the length of the modulus $(N)$ was set to 512 .

The platform for running the application was a Pentium III ( $450 \mathrm{MHz})$ processor.

\section{Experimental results}

Table 1 lists the execution time for basic operations (with a 512-bit modulus). The table does not show the time necessary for the evaluation 


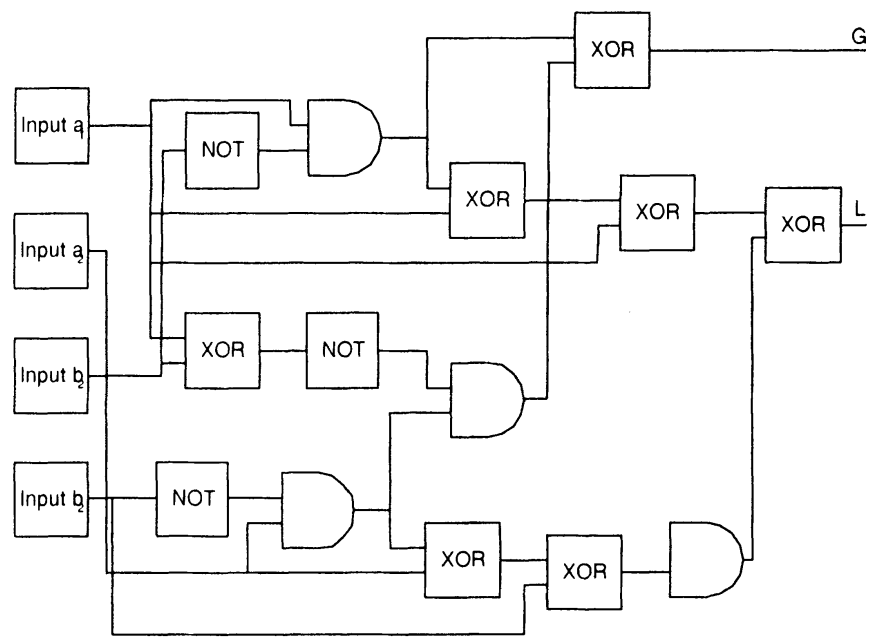

Figure 2. Boolean circuit for a two-bit comparator.

\begin{tabular}{|l|l|}
\hline Operation & Time (ms) \\
\hline Encryption of 1 bit & 65 \\
Evaluation of XOR gate & 0.28 \\
Calculation of witness & 35 \\
Usage of witness & 0.13 \\
Evaluation of NOT-gate & 0.09 \\
\hline
\end{tabular}

Table 1. Execution time for basic operations on Pentium III processor ( $450 \mathrm{MHz}$ ) with 512-bit modulus.

of an AND-gate, since it depends on the number of participants and is affected by the network latency time. The evaluation of an AND-gate requires the computation of several encryption bits and witnesses.

Table 2 lists the number of AND-gates in the boolean circuit as a function of the number of bits in the bid and the number of participants. These numbers represent about one fourth of the total number of gates in the circuit.

The total number of broadcasts can be calculated as $n .(3 a+2 b+$ $\left.1+\left\lceil\log _{2}(n+1)\right\rceil\right)$, where $n$ is the number of participants, $a$ the number of AND-gates and $b$ the number of bits in the bid. After all, every participant broadcasts its $b$ inputs, its part of the public key, 3 values per AND-gate, and $b+\left\lceil\log _{2}(n+1)\right\rceil$ witnesses for the outputs.

Not all the broadcast messages have the same size: encrypted bits have twice the size of a witness or public key. Table 3 shows the communication overhead (the total amount of bytes sent over the network) as a function of the number of bits in the bid and the number of participants. 


\begin{tabular}{|r|rrrrrrr|}
\hline $\begin{array}{c}\text { \# parti- } \\
\text { cipants }\end{array}$ & 1 & 2 & 3 & 4 & 5 & 10 & 20 \\
\hline 3 & 28 & 53 & 81 & 109 & 137 & 277 & 557 \\
4 & 60 & 108 & 162 & 216 & 270 & 540 & 1080 \\
5 & 102 & 179 & 266 & 353 & 440 & 875 & 1745 \\
6 & 158 & 270 & 397 & 524 & 651 & 1286 & 2556 \\
7 & 231 & 384 & 558 & 732 & 906 & 1776 & 3516 \\
8 & 328 & 528 & 756 & 984 & 1212 & 2352 & 4632 \\
9 & 439 & 682 & 981 & 1270 & 1559 & 3004 & 5894 \\
10 & 572 & 884 & 1241 & 1598 & 1955 & 3740 & 7310 \\
15 & 1642 & 2339 & 3141 & 3943 & 4745 & 8755 & 16775 \\
20 & 3577 & 4809 & 6231 & 7653 & 9075 & 16185 & 30405 \\
\hline
\end{tabular}

Table 2. Number of AND-gates in the complete circuit in function of the number of participants and the wordlength.

\begin{tabular}{|r|rrrrrrr}
\hline $\begin{array}{r}\text { \# parti- } \\
\text { cipants }\end{array}$ & 1 & 2 & 3 & 4 & 5 & 10 & 20 \\
\hline 3 & $28.0 \mathrm{k}$ & $52.6 \mathrm{k}$ & $80.1 \mathrm{k}$ & $107.5 \mathrm{k}$ & $135.0 \mathrm{k}$ & $272.2 \mathrm{k}$ & $546.8 \mathrm{k}$ \\
4 & $78.6 \mathrm{k}$ & $140.7 \mathrm{k}$ & $210.7 \mathrm{k}$ & $280.6 \mathrm{k}$ & $350.5 \mathrm{k}$ & $699.8 \mathrm{k}$ & $1.3 \mathrm{M}$ \\
5 & $165.5 \mathrm{k}$ & $289.6 \mathrm{k}$ & $429.7 \mathrm{k}$ & $569.8 \mathrm{k}$ & $710.1 \mathrm{k}$ & $1.3 \mathrm{M}$ & $2.7 \mathrm{M}$ \\
6 & $306.0 \mathrm{k}$ & $522.2 \mathrm{k}$ & $767.2 \mathrm{k}$ & $1012.2 \mathrm{k}$ & $1.2 \mathrm{M}$ & $2.4 \mathrm{M}$ & $4.8 \mathrm{M}$ \\
7 & $520.6 \mathrm{k}$ & $864.6 \mathrm{k}$ & $1.2 \mathrm{M}$ & $1.6 \mathrm{M}$ & $1.9 \mathrm{M}$ & $3.8 \mathrm{M}$ & $7.7 \mathrm{M}$ \\
8 & $843.7 \mathrm{k}$ & $1.3 \mathrm{M}$ & $1.8 \mathrm{M}$ & $2.4 \mathrm{M}$ & $3.0 \mathrm{M}$ & $5.8 \mathrm{M}$ & $11.6 \mathrm{M}$ \\
9 & $1.2 \mathrm{M}$ & $1.9 \mathrm{M}$ & $2.7 \mathrm{M}$ & $3.5 \mathrm{M}$ & $4.3 \mathrm{M}$ & $8.4 \mathrm{M}$ & $16.6 \mathrm{M}$ \\
10 & $1.7 \mathrm{M}$ & $2.7 \mathrm{M}$ & $3.8 \mathrm{M}$ & $5.0 \mathrm{M}$ & $6.1 \mathrm{M}$ & $11.7 \mathrm{M}$ & $22.8 \mathrm{M}$ \\
15 & $7.7 \mathrm{M}$ & $10.9 \mathrm{M}$ & $14.7 \mathrm{M}$ & $18.4 \mathrm{M}$ & $22.2 \mathrm{M}$ & $41.0 \mathrm{M}$ & $78.6 \mathrm{M}$ \\
20 & $22.3 \mathrm{M}$ & $30.0 \mathrm{M}$ & $38.9 \mathrm{M}$ & $47.8 \mathrm{M}$ & $56.7 \mathrm{M}$ & $101.2 \mathrm{M}$ & $190.1 \mathrm{M}$
\end{tabular}

Table 3. Total network overhead (in bytes) in function of the number of participants and the wordlength.

Note that the chosen modulus was 512 bits in length. A modulus of 1024 bits would double the amounts.

If there is no broadcast mechanism available, then the participants will have to use point-to-point communication. In that case, the amounts have to be multiplied by $n-1$ where $n$ is the number of participants.

The number of bits is linear with the number of bits in the bid and proportional with the cube of the number of participants (power of four without a broadcast mechanism).

\section{Discussion}

From the figures, it is clear that a secure Vickrey auction without a TTP is only feasible when the number of participants is limited. For 10 participants, $1.7 \mathrm{MB}$.. $22.8 \mathrm{MB}$ of data is sent over a broadcast network 
(17 MB .. $228 \mathrm{MB}$ when point-to-point communication is used). The number of bits in the bid can most often be kept small, since the bid might be scaled by an agreed-upon constant.

If the number of participants increases, then the auction could be run in several rounds. E.g. all participants are grouped per four, and run the protocol in each group, the winners will then bid against each other in another round. (Some modifications are necessary, since the outcome of the first round should not be decyphered and both the highest and second highest bids must be kept for the next round.)

In an Internet setting, this huge amount of communication overhead would probably prohibit the organization of such an auction. The communication overhead could be remedied by introducing semi-trusted execution sites and mobile agents. In this approach, every participant $P_{i}$ sends its representative, agent $a_{i}$, to a trusted execution site $E_{j}$. The agent contains a copy of the private keys and bid of the participant, and is capable of rumning the auction protocol. It is allowed that different participants send their agents to different sites. The only restriction being that the sites should be located closely to each other, i.e. should have high bandwidth communication between them. Of course, every execution site needs a mechanism to safely download an agent. However, that can be easily accomplished through conventional cryptographic techniques. The amount of large distance communication is moderate: every participant sends its agent to a remote site, and receives the result from its agent. The agents use a SDC-protocol, which unfortunately involves a high communication overhead. However, since the agents are executing on sites that are near each other, the overhead of the SDC-protocol is acceptable. No high bandwidth communication between the participants is necessary, and there is no longer a need for one single trusted execution site. $P_{i}$ for example, does not need to trust site $E_{k}$, used by the agent of another participant. The agents that participate in the secure computation are protected against malicious behaviour of other (non-trusted) execution sites by the SDC-protocols. That is sufficient to make this approach work.

Moreover, in contrast with the approach where one uses an unconditionally trusted third party, the trusted sites are not involved directly. They simply offer a secure execution platform: the trusted hosts do not have to know the protocol used between the agents. In other words, the combination of mobile agent technology and secure distributed computing protocols makes it possible to use generic trusted third parties that, by offering a secure execution platform, can act as trusted third party for a wide variety of protocols in a uniform way. 
Finally, the question remains whether it is realistic to assume that participants can find execution sites that are close enough to each other. Given the fact however that these execution sites can be generic, we believe that providing such execution sites could be a commercial occupation. Various deployment strategies are possible. Several service providers, each administering a set of geographically dispersed "secure hosts", can propose their subscribers an appropriate site for the secure computation. The site is chosen to be in the neighbourhood of a secure site of the other service providers involved. Another approach is to have execution parks, offering high bandwidth communication facilities, were companies can install their proprietary "secure site". The park itself could be managed by a commercial or govermment agency.

A modified version of the prototype could be used for a public "call for tender". The job would be assigned to the participant with the (second) lowest bid, without revealing the other bids. Participants can have a higher degree of confidence that their bid will be treated correctly. Colluding local authorities cannot manipulate the assignment process, neither leak bids to other parties.

One final remark about the used protocol: it requires the usage of a large modulus $N$ of which the prime factors are unknown. It seems reasonable to use a TTP for generating this modulus. However, since the TTP does not participate in the protocol otherwise, it could be an independent body that is monitored by the government and others. Organizations that would like to organize a Vickrey auction, could buy a certified modulus from this TTP.

\section{Conclusion}

Secure Vickrey auctions without a TTP are possible if the number of participants is limited. The complexity of the boolean circuit is $O\left(b . n^{2}\right)$, where $b$ is the number of bits in the bid, and $n$ the number of participants. The message complexity is $O\left(b \cdot n^{3}\right)$ if a broadcast mechanism is available, and $O\left(b . n^{4}\right)$ otherwise. The use of mobile agents might alleviate the communication overhead, by rumning the protocol on hosts that are located closely together.

\section{Acknowledgements}

The authors wish to thank Johan Daems, MSc in engineering, for building this prototype as part of his master's thesis project. The tables in this paper were extracted from [19]. 


\section{References}

[1] M. Abadi and J. Feigenbaum, "Secure circuit evaluation, a protocol based on hiding information from an oracle," Journal of Cryptology, 2(1), p. 1-12, 1990

[2] C. Cachin, J. Camenisch, J. Kilian and J. Müller, "One round Secure Computation and Secure Autonomous Mobile Agents", submitted to ICALP 2000.

[3] D. Chaum, I. Damgard and J. van de Graaf, "Multiparty computations ensuring privacy of each party's input and correctness of the result," in Advances in Cryptology-CRYPTO '87 Proceedings (Lecture Notes in Computer Science, Vol. 293), ed. C. Pomerance, p.87-119, Springer-Verlag, New York, 1988

[4] B. Chor, O. Goldreich, E. Kushilevitz and M. Sudan, "Private information retrieval," Proc. of 36th IEEE Conference on the Foundations of Computer Science (FOCS), p. 41-50, 1995

[5] R. Cramer. "An intro. secure computation", in LNCS 1561, pp 16-62, 1999.

[6] R. Cramer, I. Damgard, J. Nielsen, "Multiparty computation from Threshold Homomorphic Encryption", BRICS Report Series, ISSN 0909-0878, 2000

[7] S. Even, O. Goldreich, A. Lempel. "A randomized protocol for signing contracts." Communications of the ACM, vol. 28, 1985, pp. 637-647.

[8] M. Franklin, "Complexity and security of distributed protocols," Ph. D. thesis, Computer Science Department of Columbia University, New York, 1993

[9] M. Franklin and S. Haber, "Joint encryption and message-efficient secure computation," Journal of Cryptology, 9(4), p. 217-232, Autumn 1996

[10] O. Goldreich, S. Micali and A. Wigderson, "How to play any mental game," Proc. of 19th ACM Symposium on Theory of Computing, p. 218-229, 1987

[11] O. Goldreich, R. Vainish. "How to solve any protocol problem: an efficiency improvement", Proceedings of Crypto'87, LNCS 293, pp. 73-86, Springer, 1987

[12] S. Loureiro and R. Molva, "Privacy for Mobile Code", Proceedings of the workshop on Distributed Object Security, OOPSLA '99, p. 37-42.

[13] G. Neven, F. Piessens, B. De Decker, "On the Practical Feasibility of Secure Distributed Computing: a Case Study", to appear in Proceedings of WCC2000.

[14] G. Neven, E. Van Hoeymissen, B. De Decker, F. Piessens, "Enabling Secure Distributed Computations: Semi-trusted Hosts and Mobile Agents", Networking and Information Systems Journal, Numro spcial Mobile agents for telecommunication applications, Ed. E. Horlait, 2000, pp. 649-666

[15] N. Nisan, "Algorithms for selfish agents", Proceedings of the 16th Annual Symposium on Theoretical Aspects of Computer Science, Trier, Germany, March 1999, p. $1-15$.

[16] T. Sander and C. Tschudin, "On software protection via function hiding", Proceedings of the 2nd workshop on Information Hiding, Portland, Apr. 1998.

[17] T. Sander and C. Tschudin, "Towards mobile cryptography", Proceedings of the 1998 IEEE Symposium on Security and Privacy, Oakland, California, May 1998.

[18] T. Sander, A. Young, M. Yung, "Non-Interactive CryptoComputing for $N C^{1 "}$, preprint.

[19] J. Daems, "Veilige Vickrey veiling zonder vertrouwde derde partij", Master's Thesis, K.U.Leuven, 2001. 